\title{
Politikwechsel im Föderalismus
}

\author{
Thomas König und Thomas Bräuninger
}

\section{Politikwechsel und Vetospieler}

Die Chancen für einen Politikwechsel im deutschen Föderalismus werden als eher gering eingestuft. Anlaß zu dieser Skepsis gibt die Diagnose einer Politikverflechtung, die den kooperativen Föderalismus der Bundesrepublik charakterisieren soll. Laut Scharpf (1994: 25) führt die horizontale Politikverflechtung in eine pathologische Verflechtungsfalle von Vetospielern, da die Regierungen der unteren Länderebene nicht in der Lage sind, auf der oberen Bundesebene „einstimmige" oder "quasi-einstimmige" Entscheidungen zu treffen. Vergleichbar skeptisch fällt die Bewertung der vertikalen Politikverflechtung aus, die im Zweikammersystem von Bundestag und Bundesrat organisiert ist. Als Ursache für Entscheidungsblockaden zwischen Bundestag und Bundesrat nennen Lehmbruch (1998: 181) und Scharpf (1994: 69) die Inkongruenz von Parteienwettbewerb und Föderalismus. Besonders in Zeiten unterschiedlicher Parteimehrheiten soll die kooperative Ausrichtung des Föderalismus, dessen Arena eine Zusammenarbeit zwischen Bundestag und Bundesrat verlangt, von der kompetitiven Parteienarena überlagert werden. Nach Lehmbruchs (1998: 193) Auffassung muB daher eine losere Koppelung beider Arenen gefunden werden, wenn die Reform- und Innovationsfähigkeit des politischen Systems gesteigert werden soll.

Diese skeptische Einschätzung wird von vielen politikwissenschaflichen Untersuchungen bestätigt. In unseren bisherigen Arbeiten über das Zweikammersystem von Bundestag und Bundesrat haben wir versucht, auf notwendige und hinreichende Bedingungen für einen Politikwechsel hinzuweisen, wobei wir die Bedeutung von vier Parametern herausgestellt haben: Erstens kommt den inhaltlichen Politikvorstellungen der Akteure eine entscheidende Bedeutung zu. Dies erfordert eine empirische Erfassung und Messung der Regierungs-, Fraktions- und Länderpositionen, deren Anordnung sich womöglich von Politikfeld zu Politikfeld, in vielen Fällen sogar von Policy zu Policy unterscheidet (Bräuninger/König 1999). Zweitens besteht ein Unterschied zwischen individuellen und kollektiven Vetospielern (Tsebelis 1995; 1999; König 1997). Kollektive Vetospieler wie Bundestag und Bundesrat erlauben den mehrheitlichen Ausschluß einzelner Kammerakteure, so daß lediglich die sogenannten Pivots als die mehrheitsentscheidenden Kammerakteure in Rechnung 
des Status quo als der geltenden Regelung einbezogen werden. Je nach Lage des Status quo können sich der Agenda-Setzer und die Pivots entscheiden, ob und welche Initiative einen Politikwechsel erfolgreich herbeiführt. Ansonsten wird der Agenda-Setzer auf eine Einbringung verzichten bzw. die Pivots als Vetospieler eine Initiative ablehnen, die sie gegenüber dem Status quo schlechter stellt (König/ Bräuninger 1997).

Im folgenden Beitrag möchten wir unsere bisherigen Erkenntnisse über die Chancen für politischen Wandel im Föderalismus für die Zeit nach der Vereinigung zusammenfassen und bezüglich der Frage nach der Dynamik der Politikwechselchancen erweitern. Im ersten Kapitel werden wir auf die Bestimmung der Akteurspositionen in ausge wählten Politikfeldern eingehen. Empirisch zeigt sich für die Zeit seit der Vereinigung, da $B$ nicht nur Parteiunterschiede über die einzelnen Politikdimensionen, sondern auch über die Zeit hinweg zu beobachten sind. Ähnliches gilt für die Positionen der Regierungs- und Bundesratsakteure, deren Erfassung Konzeptions- und Meßprobleme aufwirft. Im zweiten Kapitel gehen wir auf die jeweiligen Pivots als den mehrheitsentscheidenden Akteuren in Bundestag und Bundesrat ein. Ein erster Überblick über die Mehrheitsverhältnisse in Bundestag und Bundesrat offenbart viele Veränderung seit Anfang der 90er Jahre, doch können die Pivots nur in Kombination mit den Akteurspositionen identifiziert werden. Im dritten Kapitel behandeln wir schließlich die Macht des Agenda-Setzers und bestimmen die Lage des Status quo über die Möglichkeiten, die der (vorherige) Agenda-Setzer zur Veränderung der bestehenden Regelungen hatte. Aus dieser quasi-dynamischen Betrachtung geht hervor, daß Politikwechsel trotz unterschiedlicher Mehrheitsverhältnisse möglich war und die neue Regierung Schröder insbesondere zu Beginn ihrer Amtszeit alle Chancen hatte, den von der Regierung Kohl festgelegten Status quo
zu verändern.

\section{Die Positionen der Akteure}

In der politikwissenschaftlichen Analyse kommt Akteuren, die als individuelle, korporative oder kollektive Akteure auftreten, sowie ihren Positionen eine zentrale Bedeutung für die Erklärung von Politikergebnissen zu. Ohne eine Erfassung der Positionen kann nicht geklärt werden, ob politischer Wandel ausbleibt, weil die Akteure kein Interesse an einem Politikwechsel haben oder weil das politische System Wandel ausschließt. Eine empirische Untersuchung von Akteurspositionen wirft allerdings Konzeptions- und Meßprobleme auf, da sie eine Reihe von Interessen-, Normund Regelpostulaten unterstellt, die dem Handeln der Akteure zugrunde liegen sollen. So wird für die Position eines korporativen Akteurs oftmals angenommen, da $B$ die Delegation an einen Agenten, der im Auftrag einer Organisation eine Position formuliert, für den Auftraggeber (Prinzipal) keine Risiken und Kosten aufwirft (Pappi/König/Knoke 1995). Noch problematischer erscheint die Annahme über die Eindeutigkeit einer kollektiven Akteursposition, da bei Mehrheitsentschei- den in zwei- oder mehrdimensionalen Politikräumen keine eindeutige Haltung zu erwarten ist (Hug 1999). Des weiteren stellt sich die Frage nach geeigneten Instrumenten, mit denen die individuellen, korporativen und kollektiven Akteurspositionen gemessen werden können. Während Expertenbefragungen zur Erfassung von Akteurspositionen für eine überschaubare Anzahl an Policies geeignet scheinen, existieren nur wenige Datenreihen, die einen Vergleich über die Zeit und über mehrere Politikfelder hinweg erlauben. Zur Beurteilung der Politikwechselchancen werden im folgenden die Positionen für verschiedene Akteurstypen der Zweikammergesetzgebung über einen Zeitraum von fast zehn Jahren und über vier Politikdimensionen aus den Äußerungen in Wahlprogrammen und Regierungserklärungen bestimmt.

\section{a) Parteien und Fraktionen}

Wie in jüngster Vergangenheit aus den Diskussionen zur Steuerreform bzw. zu den Bundeswehreinsätzen im Kosovo-Konflikt zu schließen war, kann die Konfiguration der Akteurspositionen von Politikdimension zu Politikdimension variieren. Während bei Bundeswehreinsätzen mittlerweile annähernd ein außenpolitischer Allparteienkonsens vorherrscht, ist die Debatte über die Steuerreform durch eine große Entfernung der wirtschaftspolitischen „linken“ und „rechten“ Parteipositionen gekennzeichnet, die bspw. von der Position einer zusätzlichen Vermögensbesteuerung bis hin zu einer drastischen Senkung des Spitzensteuersatzes reicht. Empirische Längsschnittuntersuchungen der Parteienprogrammatik haben ergeben, da $\beta$ in der Nachkriegszeit eine Polarisierung zwischen Regierungs- und Oppositionsparteien lediglich in der Wirtschaftspolitik festzustellen ist, während in gesellschafts-, umweltund außenpolitischen Fragen geringere Unterschiede zwischen den im Bundestag vertretenen Parteien existierten (Klingemann/Volkens 1997: 526ff).

Unbestreitbar ist das Parteiensystem des vereinten Deutschlands im wesentlichen das Parteiensystem der alten Bundesrepublik, was von westdeutschen Parteien forciert und den ostdeutschen Wählern bei den ersten Bundestagswahlen 1990 weitgehend akzeptiert wurde (Pappi 1994: 221). Kontrovers werden hingegen die Besonderheiten der Transformation diskutiert. Entweder wird ein „Transfer des westdeutschen Parteiensystems“ (Kaase/Gibowski 1990: 25) und eine „Adoption des bundesrepublikanischen Parteienmodells“ (Schmidt 1991) festgestellt oder das Parteiensystem im Hinblick auf die Gemeinsamkeit von Einstellungen der Wählerschaft in Ost und West als erodiert (Roth 1994; Roller 1997) bzw. mit der Etablierung der PDS als polarisiert (Veen 1995) bezeichnet. Pappi (1994: 224) will nicht ausschließen, $\mathrm{da} B$ sich das Parteiensystem vom gemäßigten Pluralismus mit vier Parteien hin zu einem polarisierten Parteiensystem nach Weimarer Art mit großen ideologischen Distanzen zwischen den Parteien entwickeln kann. 


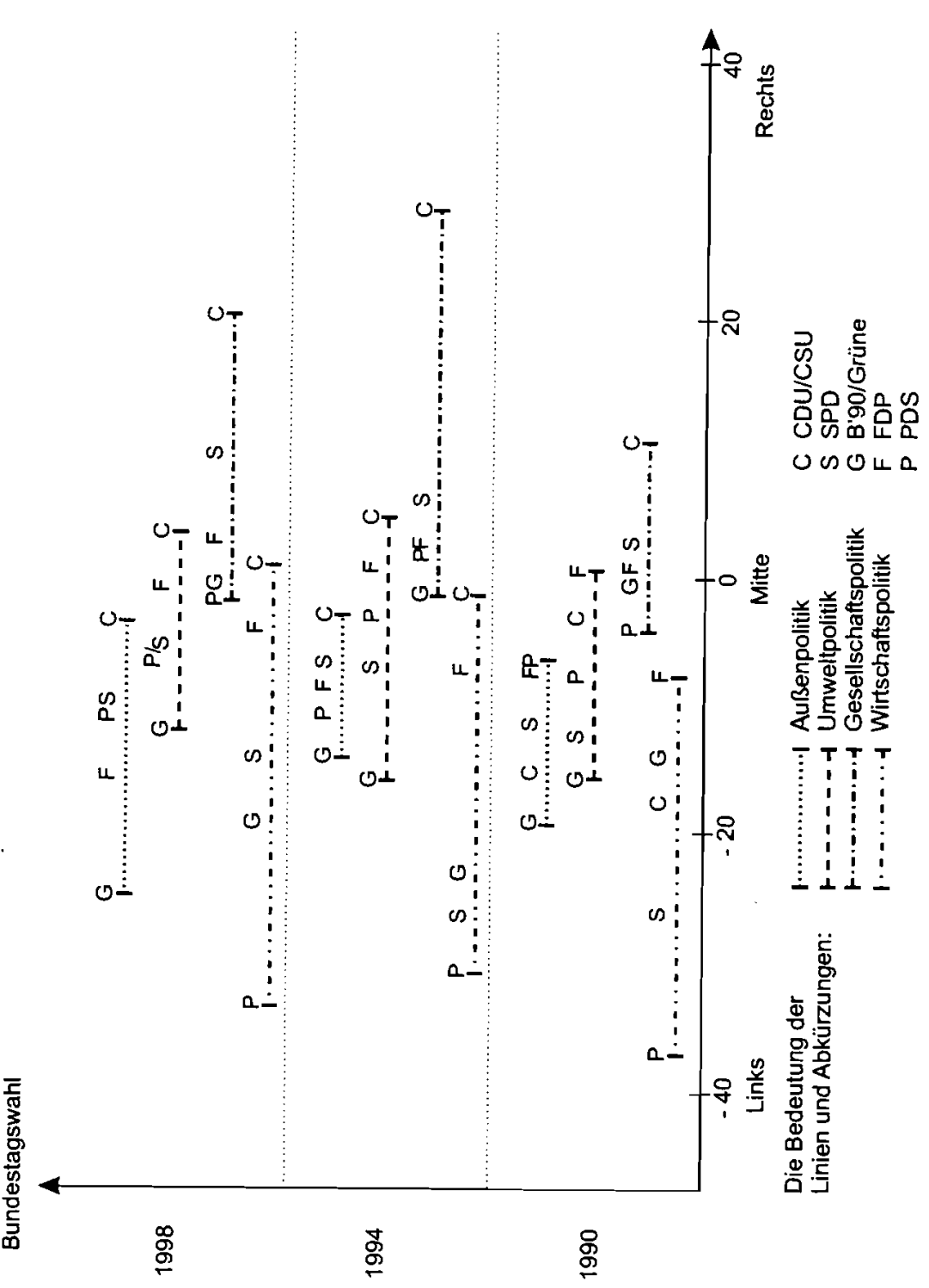

Zur Überprüfung dieser Thesen verwendet Volkens (1996: 218f.) die Wahlprogramme der Bundestagsparteien, wobei zwischen vier zentralen Politikbereichen der Wirtschafts-, Gesellschafts-, Umwelt- und Außenpolitik - unterschieden wird. Im allgemeinen führen Wahlprogramme über die nach außen gerichteten Funktionen hinaus politische Handlungsperspektiven für die Lösung von Problemen auf, die in der folgenden Legislaturperiode auf der Tagesordnung stehen (Kaack 1971: 402). Sie dienen als Grundlage für Koalitionsverhandlungen, in denen die Politik der kommenden Regierung festgelegt wird. Im Vergleich zu Koalitionsvereinbarungen, die lediglich die Regierungsparteien einschließen, geben sie Aufschluß über die Positionen im gesamten Parteienspektrum. In Abbildung 1 sind die von Volkens (1996: 219) erhobenen Daten für die Wahlprogramme dargestellt, die für die Bundestagswahl 1998 aktualisiert wurden. Im unteren Segment sind die 1990 erfaßten programmatischen Positionen der im Bundestag vertretenen Parteien zur wirtschafts-, gesellschafts-, umwelt- und außenpolitischen Dimension aufgeführt; es folgen die Segmente mit den Bundestagswahlen 1994 und 1998.

Die inhaltlichen Distanzen der Parteiakteure können aus diesen Positionen bestimmt werden, die sich aus dem prozentualen Anteil „linker“ und ,rechter“ Äußerungen zu einzelnen Themen der jeweiligen Dimension errechnen und Maximalwerte von +100 und -100 haben können (Volkens 1996: 221). Selbst auf der jeweils unten abgebildeten wirtschaftspolitischen Dimension, welche die größten Abstände zwischen den beiden extremen Parteien aufweist, ist kein Anstieg der Polarisierung zu beobachten, da der Abstand zwischen der PDS einerseits und der CDU bzw. der FDP andererseits über die Zeit hinweg relativ konstant bleibt. Lediglich auf der gesellschaftspolitischen Dimension, die an zweiter Stelle unten aufgeführt ist, schwenkt die CDU bei den Bundestagswahlen 1994 sehr stark aus. Die CDU/CSU hat sich schon in ihrem 1994er Programm in eine beachtliche konservative Richtung bewegt, was ihr nicht nur in gesellschafts-, sondern auch in wirtschaftspolitischen Fragen einen Platz am rechtskonservativen Rand des Parteienspektrums einbringt. Dadurch ist die FDP wieder in die Mitte des Parteienspektrums gerückt. In ihrem 1998er Programm verzeichnet die SPD ebenfalls einen beachtlichen programmatischen Rechtsruck, der insbesondere bei ihren Positionen zu umweltpolitischen Fragen offensichtlich wird. Neben der FDP sind B '90/Die Grünen ein relativ konstanter Faktor des Parteiensystems, wohingegen die PDS nur in wirtschaftspolitischen Fragen ihre extrem staatsorientierte Position seit der Vereinigung beibehalten hat. Insgesamt hat jedoch keine Polarisierung des Parteiensystems stattgefunden, aber einige Parteien haben in entscheidender Weise ihre Ausrichtung und somit ihre Distanzen zueinander geändert.

\section{b) Bundesregierung und Bundesrat}

Während sich die Positionen der momentan 669 Bundestagsakteure mehr oder weniger auf die Parteipositionen ihrer Fraktionen reduzieren lassen, verlangt die Erfassung der Regierungs- und 16 Bundesratsakteure weitere Messungen und Annahmen. 
Landesregierungen nach parteipolitischer Kategorisierung

\begin{tabular}{llll} 
Seit & Bundesregierung unterstützend & oppositionell & gemischt \\
\hline 11.90 & BA (C), BW (C), HE (C-F), MV (C-F), & BE (S-G), BM (S), NS (S-G), NW (S), & BB (S-F-GÜ), HA (S-F); 7 \\
$(68)$ & RP (C-F), SC (C), SN (C-F), TH (C-F); 35 & SA (S), SH (S); 26 & \\
1.91 & BA (C), BW (C), HE (C-F), MV (C-F), & BM (S), NS (S-G), NW (S), SA (S), & BE (C-S), BB (S-F-G), HA (S-F); 11 \\
$(68)$ & RP (C-F), SC (C), SN (C-F), TH (C-F); 35 & SH (S); 22 & \\
4.91 & BA (C), BW (C), MV (C-F), RP (C-F), & BM (S), HE (S-G), NS (S-G), NW (S), & BE (C-S), BB (S-F-G), HA (S-F); 11 \\
$(68)$ & SC (C), SN (C-F), TH (C-F); 31 & SA (S), SH (S); 26 & \\
5.91 & BA (C), BW (C), MV (C-F), SC (C), & BM (S), HE (S-G), NS (S-G), NW (S), & BE (C-S), BB (S-F-G), HA (S-F), RP \\
$(68)$ & SN (C-F), TH (C-F); 27 & SA (S), SH (S); 26 & (S-F); 15 \\
6.91 & BA (C), BW (C), MV (C-F), SC (C), & BM (S), HA (S), HE (S-G), NS (S-G), & BE (C-S), BB (S-F-G), RP (S-F); 12 \\
$(68)$ & SN (C-F), TH (C-F); 27 & NW (S), SA (S), SH (S); 29 & \\
12.91 & BA (C), BW (C), MV (C-F), SC (C), & HA (S), HE (S-G), NS (S-G), NW (S), & BE (C-S), BB (S-F-G), \\
$(68)$ & SN (C-F), TH (C-F); 27 & SA (S), SH (S); 26 & BM (S-F-G), RP (S-F); 15 \\
5.92 & BA (C), MV (C-F), SC (C), SN (C-F), & HA (S-STATT), HE (S-G), NS (S-G), & BE (C-S), BB (S-F-G), BM \\
$(68)$ & TH (C-F); 21 & NW (S), SA (S), SH (S); 26 & (S-F-G), BW (C-S), RP (S-F); 21 \\
7.94 & BA (C), MV (C-F), SC (C), TH (C-F); 17 & HA (S-STATT), HE (S-G), NS (S), NW (S), SA (S), BE (C-S), BB (S-F-G), BM \\
$(68)$ & & SN (S-G), SH (S); 30 & (S-F-G), BW (C-S), RP (S-F); 21 \\
9.94 & BA (C), MV (C-F), SC (C), TH (C-F); 17 & BB (S), HA (S-STATT), HE (S-G), NS (S), NW (S), BE (C-S), BM (S-F-G), \\
$(68)$ & & SA (S), SN (S-G), SH (S); 34 & BW (C-S), RP (S-F); 17 \\
10.94 & BA (C), SC (C); 10 & BB (S), HA (S-STATT), HE (S-G), NS (S), NW (S), BE (C-S), BM (S-F-G), BW (C-S), \\
$(68)$ & & SA (S), SN (S-G), SH (S); 34 & MV (C-S), RP (S-F), TH (C-S); 24 \\
5.95 & BA (C), SC (C); 10 & BB (S), HA (S-STATT), HE (S-G), NS (S), NW (S- & BE (C-S), BM (S-F-G), BW (C-S), \\
$(68)$ & & G), SA (S), SN (S-G), SH (S); 34 & MV (C-S), RP (S-F), TH (C-S); 24
\end{tabular}

$10.98 \mathrm{BB}(\mathrm{S}), \mathrm{HA}$ (S-G), HE (S-G), NS (S),

(69) NW (S-G), SA (S), SN (S), SH (S-G); 35

11.98 BB (S), HA (S-G), HE (S-G), MV (S-P),

(69) NS (S), NW (S-G), SA (S), SN (S), $\mathrm{SH}$ (S-G); 38

4.99 BB (S), HA (S-G), MV (S-P), NS (S),

(69) NW (S-G), SA (S), SN (S), SH (S-G); 33

\author{
$B B(S), H A(S-S T A T T), H E(S-G), N S(S), N W(S-B E(C-S), B M(S-C), B W(C-S), M V$ \\ G), SA (S), SN (S-G), SH (S); 34 \\ (C-S), RP (S-F), TH (C-S); 24 \\ BB (S), HA (S-STATT), HE (S-G)*, NS (S), NW \\ (S-G), SA (S), SN (S-G), SH (S); 35 \\ BE (C-S), BM (S-C), BW (C-S), MV \\ (C-S), RP (S-F), TH (C-S); 24 \\ BB (S), HA (S-STATT), HE (S-G), NS (S), NW (S- BE (C-S), BM (S-C), BW (C-S), MV \\ G), SA (S), SN (S-G), SH (S); 35 \\ (C-S), RP (S-F), TH (C-S): 24 \\ BB (S), HA (S-STATT), HE (S-G), NS (S), NW (S- BE (C-S), BM (S-C), BW (C-S), MV \\ G), SA (S), SN (S-G), SH (S-G); $35 \quad$ (C-S), RP (S-F), TH (C-S); 24 \\ $B B(S), H A(S-S T A T T), H E(S-G), N S(S), N W(S-B E(C-S), B M(S-C), M V(C-S), R P$ \\ G), SA (S), SN (S-G), SH (S-G); 35 \\ (S-F), TH (C-S); 18 \\ $B B(S), H A(S-G), H E(S-G), N S(S)$, \\ NW (S-G), SA (S), SN (S-G), SH (S-G); 35 \\ $\mathrm{BE}(\mathrm{C}-\mathrm{S}), \mathrm{BM}(\mathrm{S}-\mathrm{C}), \mathrm{MV}(\mathrm{C}-\mathrm{S}), \mathrm{RP}$ \\ (S-F), TH (C-S); 18 \\ $B B(S), H A(S-G), H E(S-G), N S(S)$, \\ NW (S-G), SA (S), SN (S), SH (S-G); 35 \\ BE (C-S), BM (S-C), MV (C-S), RP \\ (S-F), TH (C-S); 18 \\ $\mathrm{BA}(\mathrm{C}), \mathrm{BW}(\mathrm{C}-\mathrm{F}), \mathrm{SC}(\mathrm{C}) ; 16$ \\ $B E(C-S), B M(S-C), M V(C-S), R P$ \\ (S-F), TH (C-S); 18 \\ BA (C), BW (C-F), SC (C); 16 \\ $B E(C-S), B M(S-C), R P(S-F)$, \\ TH (C-S); 15 \\ BA (C), BW (C-F), SC (C), HE (C-F); 21
}

* Hessen 5 Stimmen seit 18.01.1996.

Quelle: Schindler (1994: 854), Bauer (1998: 79-83), eigene Daten.

Abkürzungen: Parteien C - CDU/CSU, F - FDP, G - B'90/Die GRÜNEN, P - PDS, S - SPD, STATT - STATT Partei Hamburg; Bundesländer (Stimmen) BA - Bayern (6), BB - Brandenburg (4), BE - Berlin (4), BM - Bremen (3), BW - Baden-Württemberg (6), HA - Hamburg (3), HE - Hessen (4/5), NS Niedersachsen (6), MV - Mecklenburg-Vorpommern (3), NW - Nordrhein-Westfalen (6), RP - Rheinland-Pfalz (4), SA - Saarland (3), SH - SchleswigHolstein (4), SN - Sachsen-Anhalt (4), SC - Sachsen (4), TH - Thüringen (4). 
Das komplexe Zustimmungsverfahren läßt sich auf die einfache Frage reduzieren, welche Mehrheiten zur Verabschiedung der Vorlage eines Agenda-Setzers notwendig sind. Betrachtet man die Verfahrensabfolge, welche die Politikinitiative einer Bundesregierung zu durchlaufen hat, dann ist die mehrheitliche Zustimmung des Bundesrats und des Bundestags erforderlich. Während im parlamentarischen System die Regierungs- und Bundestagsmehrheit bisher immer übereinstimmten, können sich die Mehrheitsverhältnisse in Bundestag und Bundesrat unterscheiden.

Zum Vergleich der Mehrheitsverhältnisse in Bundestag und Bundesrat hat sich eingebürgert, nach die Regierung unterstützende, oppositionelle und gemischte Länder zu unterscheiden (Schindler 1994; Lehmbruch 1998; König/Bräuninger 1999). Hierbei werden aus einer parteipolitischen Perspektive die Stimmen der unterstützenden Länder als Pro-Stimmen und die der oppositionellen als Kontra-Stimmen für die Bundesregierung gewertet. Besondere Bedeutung kommt bisweilen den Ländern der gemischten Kategorie zu, deren Landesregierungen Koalitionspartner vereinen, die auf Bundesebene dem Regierungs- und Oppositionslager angehören. Sie liegen zwischen den Lagern und können daher Zünglein an der Waage sein. Aus diesem Grund vereinbaren die Landesregierungen der gemischten Kategorie oftmals sogenannte Bundesratsklauseln in ihren Koalitionsverträgen, die bei Meinungsunterschieden eine Stimmenthaltung im Bundesrat vorsehen. Allerdings werden Bundesratsklauseln, die weder bindend noch einklagbar sind, bei Bedarf umgangen.

Laut Tabelle 1 konnte die Regierung Kohl nur kurzfristig mit der Unterstützung einer christlich-liberalen Bundesratsmehrheit rechnen. Von 1991 bis 1996 hatte weder das Regierungs- noch das Oppositionslager eine Parteimehrheit im Bundesrat inne, während die Opposition seit Januar 1996 die Bundesratsmehrheit besaß. Der Regierungswechsel im Herbst 1998 stellte der Regierung Schröder eine parteiliche Unterstützung durch eine sozialdemokratisch-grüne Bundesratsmehrheit in Aussicht, jedoch beendete erneut der Regierungswechsel nach den Hessenwahlen im April 1999 diese Konstellation.

\section{b) Die zustimmungspflichtigen Pivots}

Nach unserer Auffassung reicht jedoch diese Typologie zur Bestimmung der Mehrheiten in Bundesrat und Bundestag nicht aus, da die politikfeldspezifischen Vorstellungen der Akteure vernachlässigt werden. Beispielsweise lassen sich die in Abbildung 1 aufgeführten Akteurspositionen nicht auf eine allgemeine ,links-rechts'- Dimension reduzieren, die einer solchen Typologie unterliegt. Während heutzutage die PDS in wirtschafts- und gesellschaftspolitischen Fragen die extrem linke Position verfolgt, liegen B '90/Die Grünen in umwelt- und außenpolitischen Fragen an dieser Stelle. Darüber hinaus liegen CDU und SPD in außen- und gesellschaftspolitischen Fragen nahe beieinander, während größere Unterschiede zwischen beiden Parteien auf der wirtschafts- und umweltpolitischen Dimension bestehen. Vergleichbare Unterschiede finden sich auch für die Jahre zuvor, so daß eine Reduktion auf die Regierung unterstützende, oppositionelle und gemischte Länder zu kurz greifen würde.
Wir gehen vielmehr davon aus, daß die politikfeldspezifischen Pivots in Bundestag und Bundesrat über die Kombination von Mehrheitsvorgaben mit den Politikvorstellungen der Akteure zu identifizieren sind. In der Koalitionstheorie werden solche Pivots als diejenigen Akteure definiert, deren Beitritt eine Verlust- in eine Gewinnkoalition überführt. Unter Berücksichtigung der Politikvorstellungen und der Mehrheitsvorgaben nehmen diese Pivots die medianen Abstimmungspositionen auf der jeweiligen Politikdimension in Bundestag und Bundesrat ein, ohne deren Zustimmung weder „links“ noch „rechts“ eine Gewinnkoalition gebildet werden kann (Black 1958; Hinich/Munger 1997). Tabelle 2 zeigt in den Spalten 2-5 die Pivots in beiden Kammern in der Zustimmungsgesetzgebung sowie deren Positionen auf den vier Politikdimensionen. Hinsichtlich der zeitlichen Veränderungen unterscheiden wir zwischen den fünf Zeitpunkten unmittelbar vor und zu Beginn der 12., 13. und 14. Wahlperiode.

Tabelle 2: Positionen der Pivots und der Bundesregierung auf vier Politikdimensionen

\begin{tabular}{|c|c|c|c|c|c|c|c|}
\hline \multirow{2}{*}{\multicolumn{2}{|c|}{ Wirtschaftspolitik }} & \multicolumn{2}{|l|}{$\begin{array}{l}\text { Pivot im } \\
\text { Bundestag }\end{array}$} & \multicolumn{2}{|c|}{$\begin{array}{l}\text { Pivot im } \\
\text { Bundesrat }\end{array}$} & \multicolumn{2}{|l|}{ Bundesregierung } \\
\hline & & Akteur & Position & Akteur & Position & Akteur & Position \\
\hline Beginn & 12.WP & CDU/CSU & $-17,76$ & SC & $-17,76$ & CDU/CSU/FDP & $-7,23$ \\
\hline Ende & 12.WP & $\mathrm{CDU} / \mathrm{CSU}$ & $-17,76$ & BW & $-22,38$ & $\mathrm{CDU} / \mathrm{CSU} / \mathrm{FDP}$ & $-7,23$ \\
\hline Beginn & 13.WP & FDP & $-7,51$ & SN & $-27,08$ & $\mathrm{CDU} / \mathrm{CSU} / \mathrm{FDP}$ & $-2,88$ \\
\hline Ende & 13.WP & FDP & $-7,51$ & $\mathrm{RP}$ & $-18,11$ & $\mathrm{CDU} / \mathrm{CSU} / \mathrm{FDP}$ & $-2,88$ \\
\hline Beginn & 14.WP & SPD & $-14,63$ & $\mathrm{SN}$ & $-14,63$ & SPD/Grüne & $-18,99$ \\
\hline \multicolumn{8}{|c|}{ Gesellschaftspolitik } \\
\hline Beginn & 12.WP & SPD & $-0,38$ & $\mathrm{TH}$ & 6,40 & CDU/CSU/FDP & 12,45 \\
\hline Ende & 12.WP & SPD & $-0,38$ & $\mathrm{SH}$ & $-0,38$ & CDU/CSU/FDP & 12,45 \\
\hline Beginn & 13.WP & SPD & 3,63 & $\mathrm{SH}$ & 3,63 & CDU/CSU/FDP & 18,71 \\
\hline Ende & 13.WP & SPD & 3,63 & $\mathrm{SN}$ & 3,63 & CDU/CSU/FDP & 18,71 \\
\hline Beginn & 14.WP & SPD & 9,06 & SN & 9,06 & SPD/Grüne & 2,11 \\
\hline \multicolumn{8}{|c|}{ Außenpolitik } \\
\hline Beginn & 12.WP & SPD & $-12,93$ & $\mathrm{SH}$ & $-12,93$ & CDU/CSU/FDP & $-23,29$ \\
\hline Ende & 12.WP & SPD & $-12,93$ & $\mathrm{SH}$ & $-12,93$ & CDU/CSU/FDP & $-23,29$ \\
\hline Beginn & 13.WP & SPD & $-7,59$ & $\mathrm{SH}$ & $-7,59$ & CDU/CSU/FDP & $-15,11$ \\
\hline Ende & 13.WP & SPD & $-7,59$ & SN & $-7,59$ & CDU/CSU/FDP & $-15,11$ \\
\hline Beginn & 14.WP & SPD & $-10,10$ & $\mathrm{SN}$ & $-10,10$ & SPD/Grüne & $-19,41$ \\
\hline \multicolumn{8}{|c|}{ Umweltpolitik } \\
\hline Beginn & 12.WP & FDP & $-0,73$ & $\mathrm{TH}$ &,- 36 & CDU/CSU/FDP & 1,61 \\
\hline Ende & 12.WP & FDP & $-0,73$ & $\mathrm{SN}$ & $-15,62$ & CDU/CSU/FDP & 1,61 \\
\hline Beginn & 13.WP & FDP & 0,55 & $\mathrm{SH}$ & $-5,61$ & CDU/CSU/FDP & 2,88 \\
\hline Ende & 13.WP & FDP & 0,55 & $\mathrm{RP}$ & $-2,53$ & CDU/CSU/FDP & 2,88 \\
\hline Beginn & 14.WP & SPD & $-5,05$ & $\mathrm{SN}$ & $-5,05$ & SPD/Grüne & 0 \\
\hline
\end{tabular}

Abkürzungen siehe Tabelle 1. 
Beispielsweise hatte die CDU/CSU-Fraktion von 1990 bis 1994 die pivotale Bundestagsposition in der Wirtschaftspolitik inne, die 1994-98 von der FDP und mit dem Beginn der 14. Wahlperiode im Herbst 1998 von der SPD eingenommen wird. Ohne diese Akteure war im jeweiligen Zeitraum keine wirtschaftspolitische Mehrheit im Bundestag zu erzielen. Der Vergleich über die Zeit und die Politikdimensionen hinweg offenbart, daß die pivotalen Bundestagspositionen von sehr unterschiedlichen Akteuren eingenommen wurden. Zur Zeit hat die SPD alle pivotalen Positionen inne, während zuvor sowohl SPD, CDU als auch FDP diese entscheidenden Positionen einnahmen. Im Bundesrat verfügte zu Beginn der Regierung Schröder das SPD-geführte Sachsen-Anhalt auf allen vier Politikdimensionen über die entscheidende Stimme in der Zustimmungsgesetzgebung, und auch in der 13. und 14. Wahlperiode nahmen SPD-regierte Bundesländer die meisten Pivotpositionen im Bundesrat ein. Trotz unterschiedlicher Parteimehrheiten herrscht nach dieser Messung der Politikvorstellungen beispielsweise in der Außenpolitik von 1990 bis 1998 ein weitgehender Konsens zwischen Bundestag und Bundesrat, während auf den anderen Politikdimensionen größere Unterschiede zwischen Bundestags- und Bundesratspivot vorlagen.

\section{Agenda-Setzer und die Lage des Status quo}

Politische Systeme müssen Politikwechsel erlauben, um sich an neue politische $\mathrm{Be}$ dingungen adaptieren zu können. Fehlt dagegen die Möglichkeit für politischen Wandel gänzlich, dann lassen sich gesellschaftliche Entwicklungen nicht mit politischen Entscheidungen begleiten. Im extremen Fall könnten fehlende Politikwechselchancen sogar die Systemstabilität gefährden, wenn Gerichte, Bürokratien oder andere Akteure das dadurch entstehende Machtvakuum ausfüllen bzw. in die Gesetzgebung eingreifen (Tsebelis 2000). Ausgangspunkt für die Beurteilung der Politikwechselchancen ist die Wahrscheinlichkeit für ein Politikergebnis, das unter den legislativen Verfahrensvorschriften erzielbar ist.

Gemäß der Vetospielertheorie wird jedes neue Ergebnis als Abweichung von vorherigen Ergebnissen, die als Status quo bezeichnet werden, interpretiert (Tsebelis 1995). Dieser Politikwechsel wird nicht von den Pivots als den Vetospieler per se produziert. Pivots erleichtern oder erschweren lediglich Politikwechsel, der von Agenda-Setzern vorgeschlagen wird. Wichtig für die Beurteilung der Politikwechselchancen ist folglich die Interaktion zwischen Agenda-Setzer und Pivots. Für den Agenda-Setzer stellt sich grundsätzlich die Frage, ob die Pivots seiner Initiative zustimmen werden. Falls der Agenda-Setzer davon ausgeht, daß die Pivots seine Initiative ablehnen werden, dann kann er auf die Einbringung einer Vorlage verzichten. Ansonsten kann der Agenda-Setzer versuchen, seine Position durchzusetzen oder einen Kompromiß anbieten. Nach unserer Auffassung ist hierfür die Bilanz gegenüber dem Status quo entscheidend. Um diese Bilanz ziehen zu können, müssen Verhaltensannahmen für die Akteure getroffen und die Lage des Status quo bestimmt werden. a) Handlungsoptionen der Akteure

Ein Agenda-Setzer hat grundsätzlich drei Handlungsoptionen: erstens kann ein Agenda-Setzer versuchen, seine Politikposition gegenüber den Pivots durchzusetzen; zweitens kann er einen Kompromiß anbieten und drittens kann der AgendaSetzer auf die Einbringung einer Vorlage verzichten. Verzichten wird ein AgendaSetzer, wenn ihm die Einbringung einer Initiative keine Verbesserung gegenüber dem Status quo verspricht. Dieser Fall ist beispielsweise in Abbildung 2 gegeben, wenn der Agenda-Setzer den Status quo bei $s q_{0}$ nach links, der rechte Pivot jedoch nach rechts verändern möchte. Seine Vorstellungen durchsetzen kann der AgendaSetzer jedoch, wenn der Status quo eine extreme Lage, beispielsweise bei $s q_{2}$ oder $s q_{3}$, einnimmt. In diesem Fall ist die Position des Agenda-Setzers für beide pivotalen Akteure geringer entfernt, so daß mit ihrer Zustimmung zu rechnen ist. In allen anderen Fällen wird der Agenda-Setzer einen Kompromiss anbieten. Liegt die bisherige Regelung etwa bei $s q_{1}$, dann muß der Agenda-Setzer bei seinem Vorschlag insbesondere die Bilanz des Pivots auf der rechten Seite berücksichtigen.

Abbildung 2: Handlungsoptionen des Agenda-Setzers

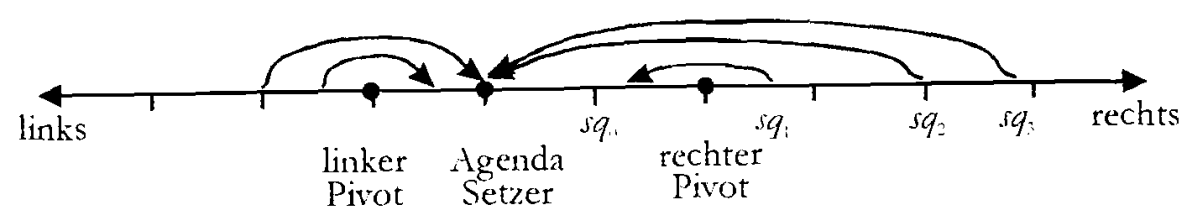

Der Agenda-Setzer hat folglich Einbringungs- und Zurückhaltungsrecht, während Pivots lediglich Vetorechte ausüben. Wir gehen davon aus, daß sie einer Vorlage zustimmen, wenn sie sich dadurch eine Verbesserung gegenüber dem Status quo erwarten. Ansonsten ist mir ihrer Ablehnung zu rechnen. Nach unserer Auffassung sind folglich nicht die parteilichen Differenzen zwischen den Akteuren als vielmehr die Bilanz ausschlaggebend, welche der Agenda-Setzer und die Pivots mit Blick auf die Veränderung des Status quo ziehen. Diese Perspektive stützen auch die Verabschiedungsquoten von Regierungsvorlagen während der Regierung Schmidt und der letzen Regierung Kohl. Trotz verschiedener Parteimehrheiten in Bundestag und Bundesrat konnten beide Regierungen Verabschiedungsrekorde aufstellen, wobei unter der letzten Regierung Kohl auch die größte Anzahl an Vorlagen seit 1949 verabschiedet wurde (König 1999).

\section{b) Eine quasi-dynamische Betrachtung des Status quo}

Für die Lage des Status quo lassen sich zwei Situationen unterscheiden. Entweder ist der Status quo beispielsweise in Form einer existierenden gesetzlichen Regelung a priori bekannt, dann läßt sich sehr einfach eine Bilanz zur Initiative des Agenda- 
Setzers ziehen. Oder der Status quo ist nicht a priori identifizierbar, da ein Regelungsbereich wie z.B. die Steuergesetzgebung sehr unterschiedliche Berührungspunkte zu existierenden Regelungen aufweist. Da in diesem Fall die Position des Status quo von der jeweiligen Initiative abhängt, ist eine allgemeingültige Voraussage über die exakte Lage des Status quo und des Vorschlags nur schwer möglich. Dennoch lassen sich auch hier Aussagen treffen, wenn anstelle einzelner, spezifischer Status quo-Positionen alle möglichen Punkte berücksichtigt werden. Hierzu müssen jedoch Annahmen über die wahrscheinliche Verteilung der Status quoPunkte getroffen werden.

Eine Annahme ist, daß in einem Politikbereich stets neue politische und gesellschaftliche Problemlagen entstehen, die der verbindlichen Regelung durch den Gesetzgeber bedürfen. Der Status quo kann in diesem Fall an allen möglichen Punkten der Politikdimension liegen, was die Annahme über eine Gleichverteilung des Status quo plausibel machen würde. Geht man von einer Gleichverteilung aus, dann bemißt die Länge des Blockade-Intervalls gerade die Wahrscheinlichkeit, mit welcher der Agenda-Setzer auf Einbringungen verzichtet. Demgegenüber wird der AgendaSetzer einen Kompromißvorschlag machen, wenn dieser den Status quo noch zu seinen Gunsten modifiziert und die Pivots zur Zustimmung bewegt. Diesen Bereich bezeichnen wir als Kompromiss-Intervall, das Politikwechsel unter Anpassung des Vorschlags an die Pivots erlaubt. Die Länge dieses Intervalls ist dann ein Maß für die Wahrscheinlichkeit der Einbringung eines Kompromißvorschlages, während die Größe des Durchsetzungsintervalls die Wahrscheinlichkeit dafür bemißt, daß der Agenda-Setzer den Status quo durch seine eigene Position ersetzen kann.

Gegen die Gleichverteilungsannahme läßt sich einwenden, daß Agenda-Setzer ihre Politikwechselchancen bereits in der Vergangenheit realisiert haben. In diesem Fall würden die Lage des Status quo und damit die aktuellen Politikwechselchancen von der Durchsetzungsfähigkeit vorheriger Agenda-Setzer abhängen. Unterliegt bspw. eine Regierung zu einem Zeitpunkt $t$ keinerlei Beschränkungen, dann wird der Status quo mit ihrer Politikvorstellung übereinstimmen. Ansonsten liegt der Status quo zum Zeitpunkt $t$ im Bereich des Blockade-Intervalls, da der Agenda-Setzer hier keine Veränderungen vornehmen kann. Für den späteren Zeitpunkt $t+1$ bedeutet dies, daß der Status quo entweder auf der Position der vorherigen Regierung oder innerhalb des ehemaligen Blockade-Intervalls liegt. Zur Beurteilung der Politikwechselchancen stellt sich bei dieser quasi-dynamischen Betrachtungsweise die Frage, ob eine neue Regierung zum Zeitpunkt $t+1$ den Status quo aus der Zeit $t$ verändern kann. Wir verwenden im folgenden dieses quasi-dynamische Modell (Krehbiel 1998: 34-47), um - ausgehend von einer Gleichverteilung des Status quo unmittelbar nach der Vereinigung - die Politikwechselchancen der Bundesregierungen zu den einzelnen, diskreten Zeitpunkten zu vergleichen.

\section{c) Die Politikwechselchancen seit der Vereinigung im Vergleich}

In den Abbildungen 3a-3d haben wir die Politikwechselchancen der Regierung Schröder aufgrund der Veränderungen des Status quo berechnet, die von den vorherigen Regierungen Kohl seit der Vereinigung auf vier Politikdimensionen vorgenommen wurden. Eingezeichnet sind die Positionen der Pivots in Bundestag und Bundesrat sowie die jeweiligen Regierungspositionen, wobei eine Unterscheidung zwischen der Situation zu Beginn und am Ende einer Legislaturperiode getroffen wird. Die beiden Akteure mit dem größten Abstand zum jeweiligen Zeitpunkt bestimmen die Lage und die Größe des Blockade-Intervalls, in dem keine Veränderung des Status quo möglich ist. So zeigen bereits die horizontal eingezeichneten Blockade-Intervalle, daß die Vorstellungen der entscheidenden Gesetzgebungsakteure in der Wirtschafts- und Gesellschaftspolitik zu fast allen Zeiten größere Differenzen aufwiesen als in der Außen- und Umweltpolitik.

Die (Nicht-)Veränderbarkeit gegebener Status quo-Lagen über die Zeit hinweg zeigt sich an den vertikal eingezeichneten Projektionslinien: Status quo-Punkte, die zum Zeitpunkt $t+1$ in das Blockade-Intervall fallen, können im Zeitraum zwischen $t$ und $t+1$ nicht verändert werden. Dies wird in der Abbildung durch senkrechte Projektionslinien dargestellt. Fallen quo-Punkte in den Bereich außerhalb des BlockadeIntervalls zum Zeitpunkt $t+1$, dann ist ein Politikwechsel möglich, den der AgendaSetzer nach seinen Vorstellungen zu gestalten versucht wird. Die schrägen Projektionslinien bilden entsprechend das Ergebnis solcher Status quo-Veränderung im Zeitraum zwischen $t$ und $t+1$ ab. Mit der Projektion über die Zeit läßt sich damit die Frage beantworten, ob Politikwechselchancen existierten, und wenn ja, zu welchem Ergebnis eine Status quo-Änderung führen müßte.

In der Wirtschaftspolitik mußte die Regierung Kohl zum Zeitpunkt der Vereinigung die Position der CDU-Fraktion im Bundestag und der CDU-geführten sächsischen Landesregierung berücksichtigen. Es bestand folglich kein Unterschied zwischen Bundestags- und Bundesratspivot, aber die Regierung konnte auch nicht uneingeschänkt den wirtschaftspolitischen Status quo verändern. Infolge Niederlagen bei anschließenden Landtagswahlen vergrößerte sich zwar der Abstand zum Bundesratspivot, dessen Position durch die Große Koalition in Baden-Württemberg repräsentiert wurde, doch veränderte dies nicht die Politikwechselchancen der Regierung Kohl. Diese blieben auch unverändert durch die Bundestagswahlen von 1994, in deren Zuge die wiedergewählte Regierung Kohl jedoch eine konservativere Wirtschaftspolitik verfolgte. Das Blockade-Intervall vergrößerte sich in dieser Legislaturperiode zum einen wegen der konservativeren Regierungshaltung, zum anderen aufgrund weiterer Verluste bei Landtagswahlen, die schließlich zu einer B-Ländermehrheit im Januar 1996 führten. Obwohl sich die Abstände zwischen der Regierung Kohl und dem Bundesratspivot seit der Vereinigung vergrößerten, hat diese Distanzierung folglich keine Auswirkungen auf die wirtschaftspolitischen Politikwechselchancen gehabt. Nach dem Wahlsieg von SPD und B`90/Die Grünen 
Abbildung 3: Politikwechselchancen

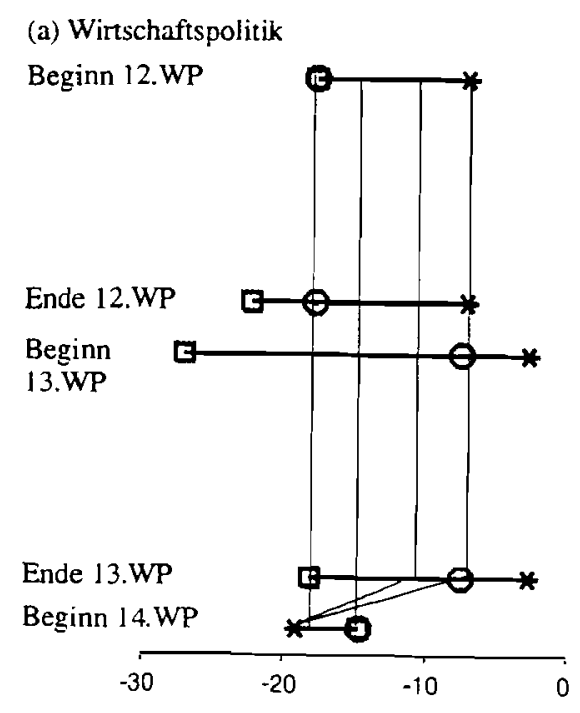

(c) Außenpolitik

Beginn 12.WP

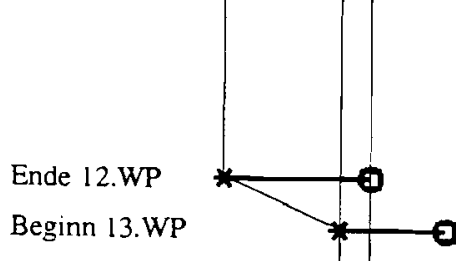

Beginn 13.WP

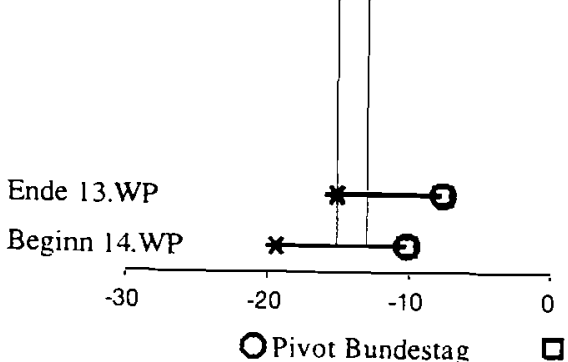

OPivot Bundestag (b) Gesellschaftspolitik

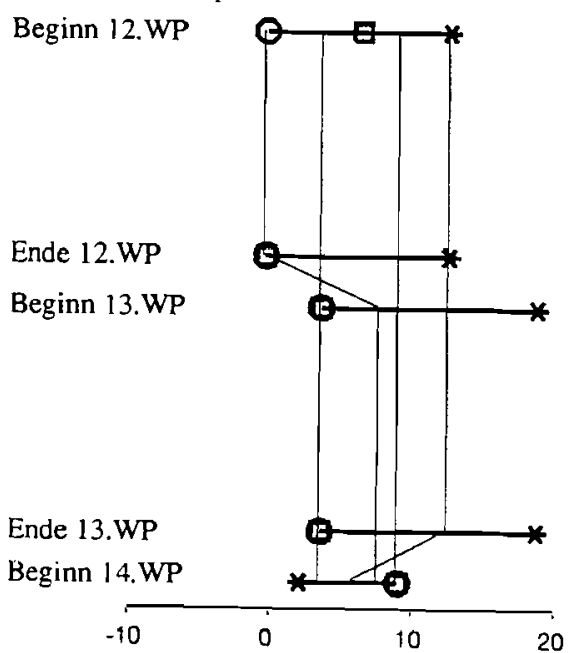

(d) Umweltpolitik

Beginn 12.WP

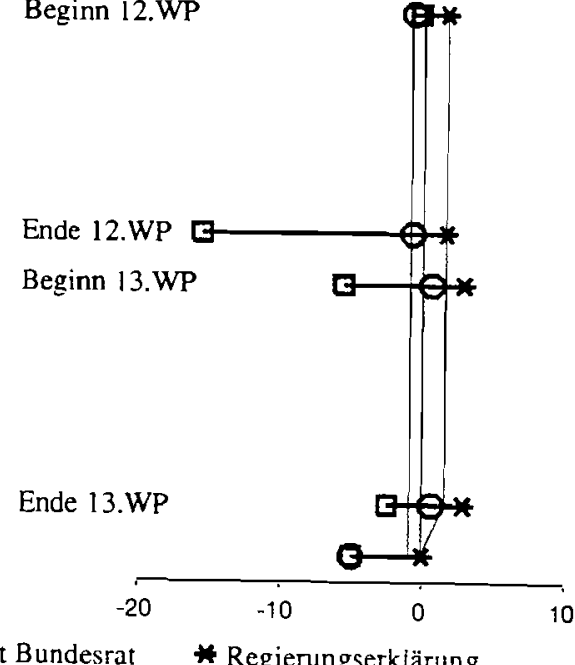

änderte sich nicht nur der wirtschaftspolitische Regierungskurs, sondern es verringerte sich auch das Blockade-Intervall zwischen Agenda-Setzer und Pivot. Die Regierung Schröder konnte nun die von den Regierungen Kohl festgelegten wirtschaftspolitischen Status quo-Punkte ändern, die ,rechts“ von der SPD lagen.

Im Vergleich zur Wirtschaftspolitik hat sich das Blockade-Intervall in der Gesellschaftspolitik seit der Vereinigung verkleinert. Der liberale FDP-Kurs ermöglichte der SPD, die gesellschaftspolitische Pivotposition im Bundestag und später auch im Bundesrat einzunehmen. Interessanterweise hat sich das Blockade-Intervall durch die konservativere SPD-Position, die sie seit der 1994er Bundestagswahl einnahm, verringert. Während die konservativere Haltung der Regierung keinen Effekt auf die Politikwechselchancen hatte, konnten nun Status quo-Punkte im „linken“ Spektrum modifiziert werden. Ein Indiz für diese Konfiguration ist die mit den SPDStimmen beschlossene Asylrechtsänderung, die trotz unterschiedlicher Parteimehrheiten in dieser Zeit verabschiedet wurde. Auch in der Gesellschaftspolitik veränderte sich mit dem 1998er Bundestagswahlergebnis die Konfiguration zwischen Pivots und Regierung: Die rot-grüne Regierung Schröder nimmt seither die linke Position ein, während die SPD und ein SPD-geführtes Land die rechte Seite des Blockade-Intervalls begrenzen, dessen Länge reduziert ist.

Während sich in der Wirtschafts- und Gesellschaftspolitik der Regierungskurs mit dem 1998er Wahlsieg von SPD und B`90/Die Grünen änderte, kennzeichnet die Umwelt- und Außenpolitik eine hohe Regierungsstabilität. In der Umweltpolitik haben sich zunächst die Politikwechselchancen gegenüber 1990 kaum verändert. Der Bereich, in dem der umweltpolitische Status quo unveränderbar blieb, wurde weder durch die 1994er Bundestagswahl noch durch Landtagswahlen beeinträchtigt. Erst die Regierung Schröder konnte die Status quo-Punkte, die zwischen ihr und den Regierungen Kohl lagen, modifizieren. Ein vergleichbares Bild findet sich für die Aussenpolitik, die bis 1994 insbesondere durch Differenzen in der Europapolitik charakterisiert war. Die Regierung Kohl verfolgte einen sehr pro-europäischen Kurs, der von dem eher zurückhaltenden SPD-Pivot begrenzt wurde. Mit dem Kurswechsel der 1994er Regierung Kohl eröffneten sich hier jedoch Politikwechselchancen, die auch nicht durch den 1998 erfolgten Regierungswechsel beeinträchtigt wurden.

Zusammenfassend zeigt sich, daß sich die Politikwechselchancen der Regierungen Kohl und Schröder über die Zeit hinweg und von Politikdimension zu Politikdimension unterscheiden. Die Wirtschaftspolitik weist bis zum rot-grünen Wahlsieg von 1998 dasselbe relativ große Blockade-Intervall auf wie auch die Umweltpolitik bis dahin kaum Veränderungen ihres relativ geringen Blockade-Intervalls erfährt. Demgegenüber eröffnet insbesondere der Wechsel des Regierungskurses in der Außenpolitik ab der 13. Wahlperiode Politikwechselchancen, die in der Gesellschaftspolitik aus der veränderten Haltung der Bundestags- und Bundesratspivots resultieren. Trotz dieser Unterschiede finden sich einige Gemeinsamkeiten. Zum einen haben die Pivots des Bundesrats die Politikwechselchancen seit der Vereinigung kaum verringert. Die Lage des Status quo der Regierung Kohl wurde in einer Zeit definiert, als die Bundesratspivots keine zusätzlichen Restriktionen aufstellten. Des 
weiteren eröffnete der Regierungswechsel von 1998 zusätzliche Chancen, den von der Regierung Kohl festgelegten Status quo zu ändern. Die Ergebnisse geben daher einen Hinweis auf die Flexibilität des Föderalismus, der auch in Zeiten verschiedener Parteimehrheiten keinen Politikwechsel ausschließt.

\section{Literatur}

Bauer, Thomas, 1998: Der Vermittlungsausschuß. Politik zwischen Konkurrenz und Konsens, Diss. Bremen.

Black, Duncan, 1958: The Theory of Committees and Elections, Cambridge.

Bräuninger, Thomas/ Thomas König, 1999: The Checks and Balances of Party Federalism. German Federal Government in a Divided Legislature, in: European Journal of Political Research 36, S. 207-235.

Hesse, Konrad/ Wolfgang Renzsch, 1990: Zehn Thesen zur Entwicklung des deutschen Föderalismus, in: Staatswissenschaften und Staatspraxis 5, S. 562-578.

Hinich, Melvin J./ Michael C. Munger, 1997: Analytical Politics, Cambridge.

Hug, Simon, 1999: Nonunitary Actors in Spatial Models. How Far is Far in Foreign Policy?, in: Journal of Conflict Resolution 43 (4), S. 479-500.

Kaack, Heino, 1971: Geschichte und Struktur des deutschen Parteiensystems, Opladen.

Kaase, Max/ Wolfgang Gibowski, 1990: Deutschland im Übergang: Parteien und Wähler vor der Bundestagswahl 1990, in: Aus Politik und Zeitgeschichte B37/38, S. 15-26.

Klingemann, Hans-Dieter/ Andrea Volkens, 1997: Struktur und Entwicklung von Wahlprogrammen in der Bundesrepublik Deutschland 1949-1994, in: Oscar Niedermayer/ Richard Stöss (Hrsg.), Parteien und Wähler im Umbruch, Opladen, S. 517-536.

König, Thomas, 1997: Politikverflechtungsfalle oder Parteienblockade? Das Potential für politischen Wandel im deutschen Zweikammersystem, in: Staatswissenschaften und Staatspraxis 2, S. 135-159.

König, Thomas, 1999: Regieren im deutschen Föderalismus, in: Aus Politik und Zeitgeschichte B13, S. 24-36.

König, Thomas/ Thomas Bräuninger, 1997: Wie wichtig sind die Länder für die Politik der Bundesregierung bei Einspruchs- und Zustimmungsgesetzen?, in: Zeitschrift für Parlamentsfragen 28, S. 605-628.

König, Thomas/ Thomas Bräuninger, 1999: A-, B- und C-Länder: Zur Verwendung des Arguments "Sprachgebrauch und benötigte Kategorie", in: Zeitschrift für Parlamentsfragen 30, S. 350-352.

König, Thomas/ Andrea Volkens/ Thomas Bräuninger, 1999: Regierungserklärungen von 1949 bis 1998. Eine vergleichende Untersuchung ihrer regierungsinternen und -externen Bestimmungsfaktoren, in: Zeitschrift für Parlamentsfragen 30, S. 641-659.

Krehbiel, Keith, 1998: Pivotal Politics: A Theory of U.S. Lawmaking, Chicago.
Lehmbruch, Gerhard, 1998: Parteienwettbewerb im Bundesstaat, Stuttgart.

Lijphart, Arend, 1984: Democracies. Patterns of Majoritarian and Consensus Government in Twenty-One Countries, New Haven.

Pappi, Franz Urban, 1994: Parteienwettbewerb im vereinten Deutschland, in: Wilhelm Bürklin/ Dieter Roth (Hrsg.), Das Superwahljahr: Deutschland vor unkalkulierbaren Regierungsmehrheiten?, Köln, S. 219-248.

Pappi, Franz U./ Thomas König/ David Knoke, 1995: Entscheidungsprozesse in der Arbeits- und Sozialpolitik. Der Zugang der Interessengruppen zum Regierungssystem über Politikfeldnetze. Ein deutsch-amerikanischer Vergleich, Frankfurt am Main.

Roller, Edeltraud, 1997: Sozialpolitische Orientierungen nach der deutschen Vereinigung, in: Oscar Gabriel (Hrsg.), Politische Entscheidungen und politisches Verhalten im TransformationsprozeB, Opladen, S. 115-146.

Roth, Dieter, 1994: Was bewegt die Wähler?, in: Aus Politik und Zeitgeschichte B21, S. 3-13.

Sartori, Giovanni, 1992: Demokratietheorie, Darmstadt.

Scharpf, Fritz W., 1994: Optionen des Föderalismus in Deutschland, Frankfurt am Main.

Schmidt, Manfred, 1991: Machtwechsel in der Bundesrepublik (1949-1990), in: Bernhard Blanke/ Helmut Wollmann (Hrsg.), Die alte Bundesrepublik. Kontinuität und Wandel, Opladen, S. 179-203.

Schindler, Peter, 1994: Datenhandbuch zur Geschichte des Deutschen Bundestages 1983 bis 1991, Baden-Baden.

Shepsle, Kenneth A./ Barry R. Weingast, 1987: The Institutional Foundations of Committee Power, in: American Political Science Review 81, S. 85-104.

Tsebelis, George, 1995: Decision Making in Political Systems: Veto Players in Presidentialism, Parliamentarism, Multicameralism und Multipartyism, in: British Journal of Political Science 25, S. 289-325.

Tsebelis, George, 1999: Veto Players and Law Production in Parliamentary Democracies: An Empirical Analysis, in: American Political Science Review 93, S. 591-605.

Tsebelis, George, 2000: Veto Players and Institutional Analysis, in: Governance (im Erscheinen).

Tsebelis, George/ Jeannette Money, 1997: Bicameralism, Cambridge.

Veen, Hans-Joachim, 1995: Zwischen Rekonzentration und neuer Diversifizierung. Tendenzen der Parteientwicklung fünf Jahre nach der deutschen Einheit, in: Winand Gellner/ Hans-Joachim Veen (Hrsg.), Umbruch und Wandel in westeuropäischen Parteiensystemen, Frankfurt am Main, S. 117-133.

Volkens, Andrea 1996: Parteiprogramme und Polarisierung, in: Oskar Niedermayer (Hrsg.), Intermediäre Strukturen in Ostdeutschland, Opladen, S. 215-236. 